\title{
Pacific
}

Journal of

Mathematics

\section{ON THE MAPPING INTERSECTION PROBLEM}

\section{ALEXANDER DRANISHNIKOV}

Volume $173 \quad$ No. 2 


\title{
ON THE MAPPING INTERSECTION PROBLEM
}

\author{
A.N. DRANishnikov
}

It is proved that if the inequality $\operatorname{dim} X \times Y<n$ holds for compacta $X$ and $Y$ with $\operatorname{dim} X$ or $\operatorname{dim} Y \neq n-2$ then for every pair of maps $f: X \rightarrow \mathbb{R}^{n}$ and $g: Y \rightarrow \mathbb{R}^{n}$ and for any $\epsilon>0$ there are $\epsilon$-close maps $f^{\prime}: X \rightarrow \mathbb{R}^{n}$ and $g^{\prime}: Y \rightarrow \mathbb{R}^{n}$ with $f^{\prime}(X) \cap g^{\prime}(Y)=\oslash$. Thus an affirmative answer to the Mapping Intersection Problem is given except in the codimension two case. The solution is based on previous results in this subject and on a generalization of the Eilenberg Theorem.

\section{Introduction.}

We say two compacta $X$ and $Y$ have the unstable intersection property in Euclidean space $\mathbb{R}^{n}$ (and denote it by $X \| Y$ ) if every pair of maps $f: X \rightarrow \mathbb{R}^{n}$ and $g: Y \rightarrow \mathbb{R}^{n}$ can be approximated arbitrarily closely by maps $f^{\prime}: X \rightarrow \mathbb{R}^{n}$ and $g^{\prime}: Y \rightarrow \mathbb{R}^{n}$ with disjoint images $\left(\operatorname{Im} f^{\prime} \cap \operatorname{Im} g^{\prime}\right)=\oslash$.

If $X$ and $Y$ are polyhedra then $X \| Y$ in $\mathbb{R}^{n}$ if and only if $\operatorname{dim} X+\operatorname{dim} Y<n$. This is the so-called general position property. If one of $X, Y$ is a polyhedron, again there is an equivalence $X \| Y \Longleftrightarrow \operatorname{dim} X+\operatorname{dim} Y<n$. An interest in the property $X \| Y$ for arbitrary compacta arose after McCullough's and Rubin's paper [1], where an example of an n-dimensional compactum $X$ is constructed with $X \| X$ in $\mathbb{R}^{2 n}$. Their compactum $X$ had the property $\operatorname{dim} X \times X<2 n$. Then the natural conjecture appeared: $X \| Y$ in $\mathbb{R}^{n}$ if and only if $\operatorname{dim} X \times Y<n$.

The conjecture was immediately proved in the complementary case: $\operatorname{dim} X+$ $\operatorname{dim} Y=n[\mathbf{2}],[\mathbf{3}],[\mathbf{4}],[\mathbf{5}],[\mathbf{6}]$. It was known from [7] that the inequality $\operatorname{dim} X \times Y<n$ gives only one restriction $\operatorname{dim} X+\operatorname{dim} Y \leq 2 n-4$ on the sum of the dimensions, which is far beyond the complementary case.

The proof in [6] in the complementary case is based on Alexander duality. That proof was extended later to the metastable case by means of Spanier-Whitehead duality in [8],[9]. A different approach based on Weber's theorem was used in [10].

The author considered the following parallel problem: Suppose that the compactum $X$ is a subset of $\mathbb{R}^{n}$. Under what conditions can every map $f: Y \rightarrow \mathbb{R}^{n}$ be approximated by a map $f^{\prime}: Y \rightarrow \mathbb{R}^{n}-X$ avoiding $X ?$ Such a subset $X \subset \mathbb{R}^{n}$ is called $Y$-negligible. In $[11],[12]$ this problem was solved for tame $X$ with $\operatorname{codim} X \neq 2$ : 
The Negligibility Criterion. Suppose $X$ is a tame compactum in $\mathbb{R}^{n}$ of $d i$ mension $\operatorname{dim} X \neq n-2$. Then $X$ is $Y$-negligible if and only if $\operatorname{dim} X \times Y<n$.

Soon after, the conjecture was proved in the direction " $X \| Y$ in $\mathbb{R}^{n} \Rightarrow \operatorname{dim} X \times Y<$ $n "[\mathbf{1 3}]$.

A significant move toward the conjecture was made in [14]. There the conjecture was proved for the case $\operatorname{codim} X \cdot \operatorname{codim} Y \geq n$ (except the $\operatorname{codim}=2$ case). Moreover the remaining part of the conjecture " $\operatorname{dim} X \times Y<n \Rightarrow X \| Y$ in $\mathbb{R}^{n}$ " which is called the Mapping Intersection Problem, was reduced to the Embedding Problem for cohomological dimension: given a compactum $X$ of dimension $\leq n-2$, does there exist a compactum $X^{\prime} \subset \mathbb{R}^{n}$ such that $c$ - $\operatorname{dim}_{G} X=c-\operatorname{dim}_{G} X^{\prime}$ for all abelian groups $G$ ? We say in that case that $X$ and $X^{\prime}$ have the same cohomological dimension type. Even more, it was proved in [14] that the Mapping Intersection Problem is equivalent to the Embedding Problem.

According to the Splitting Theorem ([14]), every $n$-dimensional compactum has the cohomological dimension type of a compact countable wedge of fundamental compacta $\bigvee_{i=1}^{\infty} F\left(G, n_{i}\right)$ for $n_{i} \leq n$. The cohomological dimensions of the fundamental compacta are defined by the Kuz'minov table [15], [7], [14]

\begin{tabular}{|c|c|l|l|l|l|l|c|}
\hline \multicolumn{2}{|c|}{ dimensions of $F(G, n)$} & \multicolumn{1}{|c|}{} \\
\hline compacta & $\mathbb{Z}_{(p)}$ & $\mathbb{Z}_{p}$ & $\mathbb{Z}_{p^{\infty}}$ & $\mathbb{Q}$ & $\mathbb{Z}_{(q)}$ & $\mathbb{Z}_{q}$ & $\mathbb{Z}_{q^{\infty}}$ \\
\hline$F(\mathbb{Q}, n)$ & $\mathrm{n}$ & 1 & 1 & $\mathrm{n}$ & $\mathrm{n}$ & 1 & 1 \\
\hline$F\left(\mathbb{Z}_{(p)}, n\right)$ & $\mathrm{n}$ & $\mathrm{n}$ & $\mathrm{n}$ & $\mathrm{n}$ & $\mathrm{n}$ & 1 & 1 \\
\hline$F\left(\mathbb{Z}_{p}, n\right)$ & $\mathrm{n}$ & $\mathrm{n}$ & $\mathrm{n}-1$ & 1 & 1 & 1 & 1 \\
\hline$F\left(\mathbb{Z}_{p^{\infty}}, n\right)$ & $\mathrm{n}$ & $\mathrm{n}-1$ & $\mathrm{n}-1$ & 1 & 1 & 1 & 1 \\
\hline
\end{tabular}

Here $p, q$ are primes, $q$ runs over all primes $\neq p$ and $\mathbb{Q}$ is the rationals, $\mathbb{Z}_{(p)}$ is the localization of the integers at $p, \mathbb{Z}_{p}=\mathbb{Z} / p \mathbb{Z} ; \mathbb{Z}_{p^{\infty}}=\mathbb{Q} / \mathbb{Z}_{(p)}$.

In this paper we prove that all $n$-dimensional fundamental compacta $F(G, n)$ can be realized in $\mathbb{R}^{n+2}$. Therefore the Embedding Problem for cohomological dimension has an affirmative answer by virtue of the Splitting Theorem. The proof is based on a generalization of the following Eilenberg theorem [22]: Suppose that $f: A \rightarrow S^{k}$ is a map of a closed subset $A \subset X$ of a compactum $X$ of dimension $\operatorname{dim} X \leq n+1$ to the $k$-dimensional sphere. Then there exists a compactum $Y \subset X$ of dimension $\operatorname{dim} Y \leq n-k$ such that $f$ has an extension over $X-Y$.

We recall that the inequality $\operatorname{dim} X \leq m$ is equivalent to the following property

$X \tau S^{m}$ : for any map $f: A \rightarrow S^{m}$ of a closed subset $A \subset X$, there is an extension $\bar{f}: X \rightarrow S^{m}$. The other notation for this is $S^{m} \in A E(X)$ i.e. the sphere $S^{m}$ is an absolute extensor for the class $\{X\}$ consisting of one space $X$.

So, there is the following way to generalize Eilenberg's theorem: replace the condition $\operatorname{dim} X \leq n+1 \Longleftrightarrow X \tau\left(S^{k} * S^{n-k}\right)$ by $X \tau\left(S^{k} * L\right)$ and $\operatorname{dim} Y \leq n-k$ by 
$Y \tau L$. Moreover, we may consider instead of the $k$-sphere $S^{k}$ an arbitrary complex $K$. The corresponding generalization for countable CW-complexes $K$ and $L$ is proved in $\S 2$.

As a consequence the Generalized Eilenberg theorem yields the solution for countable complexes $K$ and $L$ of the problem from [16]: If a compact space $X$ has the property $X \tau(K * L)$ then $X$ is the union $X=Z \cup(X-Z)$, where $Z \tau K$ and $(X-Z) \tau L$.

Thus, the Mapping Intersection Problem is completely solved except in the codimension two case. The problem here is to prove The Negligibility Criterion for compacta of codimension two.

\section{The Generalized Eilenberg theorem.}

By $K * L$ we denote the join product of spaces $K$ and $L: K * L \simeq K \times L \times$ $[-1,1] /(x, y,-1) \sim\left(x, y^{\prime},-1\right) ;(x, y, 1) \sim\left(x^{\prime}, y, 1\right)$. There are natural imbeddings $K \subset K * L$ and $L \subset K * L$. Denote by $\pi_{K}: K * L-L \rightarrow K$ and $\pi_{L}: K * L-K \rightarrow L$ the natural projections.

It's known that the quotient topology on $K * L$ is not the appropriate one for the general type of CW-complexes, but for locally compact complexes it's good. Since for the purpose of this paper it's sufficient to consider only locally compact CW-complexes, we will not discuss the topology on the join $K * L$ as well as on the other constructions like the product $K \times L$ and the smash product $K \wedge L$.

Proposition 2.1. Let a space $X=A \cup B$ be the union of closed subsets and let $f: A \rightarrow K$ and $g: B \rightarrow L$ be maps to absolute neighborhood extensors $K$ and $L$. Then there exists a map $\psi: X \rightarrow K * L$ with the properties $\left.\pi_{K} \circ \psi\right|_{A}=f$ and $\left.\pi_{L} \circ \psi\right|_{B}=g$.

Proof. First, we extend $f$ and $g$ over some open neighbourhoods to $f^{\prime}: U \rightarrow K$ and $g^{\prime}: V \rightarrow L, A \subset U, B \subset V$. Let us consider a function $\phi: X \rightarrow[-1,1]$ such that $\phi^{-1}(-1)=A-V$ and $\phi^{-1}(1)=B-U$. We define

$$
\psi(x)= \begin{cases}\left(f^{\prime}(x), g^{\prime}(x), \phi(x)\right), & \text { if } x \in U \cap V \\ (f(x), *,-1), & \text { if } x \in A-V \\ (*, g(x), 1), & \text { if } x \in B-U\end{cases}
$$

Proposition 2.2. Let $X$ be a compact metric space and let $K$ be a countable $C W$-complex. Then there exists a countable family $\left\{f_{i}: B_{i} \rightarrow K\right\}$ of maps of closed subsets of $X$ such that for any closed $B \subset X$ and any map $f: B \rightarrow K$ there is a 
number $i$ for which $B \subset B_{i}$ and the restriction $\left.f_{i}\right|_{B}$ is homotopic to $f$.

Proof. Denote by $C l(B)$ the closure of a subset $B \subset X$. Consider a countable basis $\left\{U_{j}\right\}_{j \in N}$ for the topology of $X$ and for every finite subset $\alpha \subset \mathbb{N}$ define $B_{\alpha}=\bigcup_{j \in \alpha} C l\left(U_{j}\right)$. Then for every $\alpha$ we consider the set $\left[B_{\alpha}, K\right]$ of homotopy classes. Since $B_{\alpha}$ is compact and the complex $K$ is countable, the set $\left[B_{\alpha}, K\right]$ is countable. For every $\alpha$ choose a countable family $\left\{f_{j}^{\alpha}: B_{\alpha} \rightarrow K\right\}$ of representatives and then renumerate the family $\left\{f_{j}^{\alpha}\right\}$ to obtain $\left\{f_{i}: B_{i} \rightarrow K\right\}_{i \in N}$.

Let $B$ and $f: B \rightarrow K$ be arbitrary. There is an extension $\bar{f}: U \rightarrow K$ over an open neighbourhood $U$. Since $\left\{U_{j}\right\}_{j \in N}$ is a basis and $B$ is compact, there is an $\alpha$ such that $B \subset B_{\alpha} \subset U$. The restriction $\left.\bar{f}\right|_{B_{\alpha}}$ is homotopic to some $f_{i}: B_{i}=B_{\alpha} \rightarrow K$. Hence $\left.f_{i}\right|_{B}$ is homotopic to $f$.

Note that every countable CW-complex is homotopy equivalent to a locally compact complex. Since the extension property $X \tau K$ depends only on the homotopy type of $K$, we will assume that all our countable complexes are also locally compact.

Theorem 1. Suppose that $K$ and $L$ are countable $C W$-complexes and $X$ is a compact metric space with the property $X \tau K * L$. Let $g: A \rightarrow K$ be a continuous map of a closed subset $A \subset X$. Then there exists a compact set $Y \subset X$ having the property $Y \tau L$ and the map $g$ is extendable over $X-Y$.

Proof. Let $\left\{f_{i}: B_{i} \rightarrow L\right\}$ be a family for $X$ as in Proposition 2.2. By induction we construct a sequence $g_{i}: A_{i} \rightarrow K$ such that for all $i A_{i} \subset \operatorname{Int} A_{i+1},\left.g_{i+1}\right|_{A_{i}}=g_{i}$, $A_{1}=A, g_{1}=g$ and $f_{i}$ is extendable over $X-A_{i+1}$. Then the set $\bigcup_{i} A_{i}$ is open and we can define $Y=X-\bigcup_{i} A_{i}$. The union $\cup g_{i}$ gives an extension of $g$ over $X-Y=\bigcup_{i} A_{i}$. Clearly $Y \tau L$. Indeed, consider an arbitrary map $f: B \rightarrow L$, $(B \subset Y)$; then there exists $i$ such that $B \subset B_{i}$ and $\left.f_{i}\right|_{B}$ is homotopic to $f$. Since $f_{i}$ is extendable over $Y$, the Homotopy Extension Theorem implies that $f$ is also extendable over $Y$.

We define $A_{1}=A$ and $g_{1}=g$.

Assume that the sequences $A_{1} \subset \operatorname{Int} A_{2} \subset \ldots \subset A_{n}$ and $\left\{g_{i}: A_{i} \rightarrow K\right\}$ are constructed such that $\left.g_{i+1}\right|_{A_{i}}=g_{i}$ and $f_{i}$ is extendable over $X-A_{i+1}$ for $i<n$.

By means of $g_{n}$ and $f_{n}$ we define a map $\psi_{n}: A_{n} \cup B_{n} \rightarrow K * L$ as in 2.1. Since $X \tau K * L$, there is an extension $\bar{\psi}_{n}: X \rightarrow K * L$. Let $U_{n}$ be an open neighbourhood of $\bar{\psi}_{n}^{-1}(L)$ in $\bar{\psi}_{n}^{-1}(K * L-K)$ such that $C l\left(U_{n}\right) \subset X-A_{n}$. We define $A_{n+1}=X-U_{n}$ and $g_{n+1}=\left.\pi_{K} \circ \bar{\psi}_{n}\right|_{A_{n+1}}$. It is clear that $f_{n}$ is extendable over $\bar{\psi}_{n}^{-1}(K * L-K)$ and, hence, over $U_{n}=X-A_{n+1}$. By 2.1 we have $\left.g_{n+1}\right|_{A_{n}}=g_{n}$.

A complex $L$ has the completion property if every $\sigma$-compact metric space $Z$ with the property $Z \tau L$ has a completion $\bar{Z}$ with the same property. It is known that the Eilenberg-MacLane space $K\left(\mathbb{Z}_{p}, n\right)$ has the completion property for finite dimensional $Z$ [15], [17]. The proof of that fact is valid for arbitrary $L$ with finite 
skeletons $L^{(k)}$ for all $k$. Other results on the completion property are in [16], and the most recent result on that subject is due to Olszewski and it states that every countable CW-complex has the completion property [25].

Corollary 2. Suppose that $K$ and $L$ are countable complexes. If for some compactum $X$ the property $X \tau K * L$ holds then there is a $G_{\delta}$-set $Z \subset X$ such that $Z \tau L$ and $(X-Z) \tau K$.

Proof. According to 2.2 there is a countable family $\left\{g_{i}: A_{i} \rightarrow K\right\}$ such that any map $g: A \rightarrow K$ of a closed subset $A$ of $X$ is homotopic to some restriction $\left.g_{i}\right|_{A}$. By Theorem 1 there exists a sequence of compacta $Y_{i} \subset X$ such that $Y_{i} \tau L$ and $g_{i}$ is extendable over $X-Y_{i}$. By the countable union theorem (the next proposition) we have the property $\left(\bigcup Y_{i}\right) \tau L$. By the completion property of $L$ there is a $G_{\delta^{-}}$ set $Z \subset X, Z \supset \cup Y_{i}$ and $Z \tau L$. Now consider the complement $X-Z$. By the construction every compact subset $Y \subset X-Z$ has the property $Y \tau K$. The countable union theorem implies $(X-Z) \tau K$.

Proposition 2.3. (Countable union theorem). Suppose that $K$ is a $C W$-complex. Let $X$ be a metrizable space and $X=\bigcup X_{i}$ where each $X_{i}$ is closed in $X$ and has the property $X_{i} \tau K$. Then $X \tau K$.

Proof. Let $f: A \rightarrow K$ be an arbitrary map. By induction we construct a sequence $f_{i}: A_{i} \rightarrow K$ such that $A_{i}$ is closed and $A_{i} \subset \operatorname{Int} A_{i+1},\left.f_{i+1}\right|_{A_{i}}=f_{i}$ and $X=\bigcup A_{i}$. That would be sufficient to get an extension $\bar{f}: X \rightarrow K$.

Let $A_{1}=A$ and $f_{1}=f$. Assume that $f_{n}: A_{n} \rightarrow K$ is constructed. Since $X_{n} \tau K$ there is an extension $\bar{f}_{n}: A_{n} \cup X_{n} \rightarrow K$. There is an extension $f_{n+1}$ of $\bar{f}_{n}$ over a closed neighbourhood $A_{n+1}$ containing $A_{n} \cup X_{n}$

\section{Realization of Fundamental compacta in Euclidean space.}

The cohomological dimension of a topological space $X$ is the highest number $n$ such that for some closed subset $A \subset X$, the $n$-dimensional cohomology group $\check{H}^{n}(X, A ; G)$ is non-trivial. We denote it by $\mathrm{c}-\operatorname{dim}_{G} X$, where $G$ is a coefficient group. It is known [15], [7] that the inequality $c-\operatorname{dim}_{G} X \leq n$ is equivalent to the property $X \tau K(G, n)$ where $K(G, n)$ is the Eilenberg-MacLane complex (here $n \geq 1$ and $X$ is at least paracompact).

By $M(G, n)$ we denote the Moore space, i.e., $M(G, n)$ is a CW-complex with trivial homology groups in dimensions $i \neq n$ and with $H_{n}(M(G, n))=G$.

Lemma 3.1. Suppose that $L * M(G, 1)$ is $(n+1)$-connected for some countable complex $L$ and for some abelian group $G$. Then there exist an $n$-dimensional compactum $Y \subset \mathbb{R}^{n+2}$ with non-trivial Steenrod homology group $H_{n}(Y ; G) \neq 0$ and with 
$Y \tau L$

Proof. Let $A=S^{1} \subset S^{n+2}$ be a circle in the $n+2$-dimensional sphere and let $g: A \rightarrow M(G, 1)$ induce a non-trivial element of $\pi_{1}(M(G, 1))$. By Theorem 2 there exist a compactum $Y \subset S^{n+2}$ with $Y \tau L$ and an extension $\bar{g}: S^{n+2}-Y \rightarrow$ $M(G, 1)$. Since the natural inclusion $i: M(G, 1) \rightarrow K(G, 1)$ induces an isomorphism of the fundamental groups, the composition $i \circ g$ is a homotopically non-trivial map. Therefore $i \circ \bar{g}$ is a homotopically non-trivial map. The map $i \circ \bar{g}$ represents some non-trivial element $\alpha \in \check{H}^{1}\left(S^{n+2}-Y ; G\right)$. By Sitnikov duality [23] there is a dual non-trivial element $\beta \in H_{n}(Y ; G)$. This implies that $\operatorname{dim} Y \geq n$. We always may assume that $\operatorname{dim} Y=n$.

Lemma 3.2. Suppose that two countable abelian groups have the properties $H \otimes$ $G=0$ and $\operatorname{Tor}(H, G)=0$ (Tor means the torsion product [24]). Then for every $n$ there exists an $n$-dimensional compactum $Y \subset \mathbb{R}^{n+2}$ with $c-\operatorname{dim}_{H} Y \leq 1$ and $H_{n}(Y ; G) \neq 0$.

Proof. For any pair of locally compact based spaces $\left(X, x_{0}\right)$ and $\left(Y, y_{0}\right)$ there is a closed contractible set $C$ lying in $X * Y$ such that the quotient space $X * Y / C$ is homeomorphic to the reduced suspension over the smash product $X \wedge Y$. Indeed, define $C$ as the union of the cones above $X$ and $Y$ naturally embedded in $X * Y$. Thus for locally compact CW-complexes $X$ and $Y$ we have the equality $X * Y=\Sigma(X \wedge Y)$ of homotopy types. Since the Moore spaces $M(G, 1)$ and $M(H, 1)$ can be represented by locally compact CW-complexes, we may compute homology groups $H_{i}(M(H, 1)$ * $M(G, 1))$ via homology groups of the smash product. The homology group of the smash product $X \wedge Y$ is equal to the homology group of the pair $(X \times Y, X \vee Y)$. Now the homology exact sequence of the pair $(M(H, 1) \times M(G, 1), M(H, 1) \vee M(G, 1))$ and the Kunneth formula imply that $H_{i}(M(H, 1) * M(G, 1))=0$ for all $i>0$. Since $\pi_{1}(M(H, 1) * M(G, 1))=0$, the space $M(H, 1) * M(G, 1)$ is $n$-connected for all $n$ by the Hurewicz theorem. Lemma 3.1 yields a $Y \subset S^{n+2}$ with $Y \tau M(H, 1)$. By Theorem 6 of $[18]$ the property $Y \tau M$ implies the property $Y \tau S P^{\infty} M$ where $S P^{\infty}$ is the infinite symmetric power. According to the Dold-Thom theorem [19] $S P^{\infty} M(H, 1)=K(H, 1)$.

So, we have the property $Y \tau K(H, 1)$ and hence $c-\operatorname{dim}_{H} Y \leq 1$.

Theorem 3. For every $n$ there are $n$-dimensional fundamental compacta $F(G, n)$ lying in $\mathbb{R}^{n+2}$.

Proof. We have four series of fundamental compacta. So, let us consider four cases.

1) $F(\mathbb{Q}, n)$. We define $H=\bigoplus_{\text {all } p} \mathbb{Z}_{p}$ and $G=\mathbb{Q}$. Then the properties $G \otimes H=$ $\operatorname{Tor}(G, H)=0$ hold. Apply Lemma 3.2 to obtain an $n$-dimensional compactum $Y \subset$ $\mathbb{R}^{n+2}$ with c- $\operatorname{dim}_{H} Y \leq 1$. Then it follows that c-dim $\mathbb{Z}_{p} Y \leq 1$ for all primes $p$. The Bokstein inequality c-dim $\mathbb{Z}_{p} \geq c$ - $\operatorname{dim}_{\mathbb{Z}_{p} \infty}[15],[7]$ implies that c-dim $\mathbb{Z}_{\mathbb{Z}^{\infty}} Y \leq 1$. The 
other Bokstein inequality c-dim $\mathbb{Z}_{(p)} Y \leq \max \left\{c-\operatorname{dim}_{\mathbb{Q}} Y, c-\operatorname{dim}_{\mathbb{Z}_{p \infty}} Y+1\right\}$ implies c$\operatorname{dim}_{\mathbb{Z}_{(p)}} Y \leq \mathrm{c}-\operatorname{dim}_{\mathbb{Q}} Y$ provided c-dim $\mathbb{Q} Y \geq 2$. According to Lemma $3.2 H_{n}(Y, \mathbb{Q}) \neq$ 0 and hence $c-\operatorname{dim}_{\mathbb{Q}} Y \geq n \geq 2$.

Since $Y$ is $n$-dimensional, $c-\operatorname{dim}_{\mathbb{Q}} Y \leq n$ and hence c- $\operatorname{dim}_{\mathbb{Q}} Y=n$. The Bokstein inequality c-dim ${ }_{\mathbb{Q}} \leq \mathrm{c}-\operatorname{dim}_{\mathbb{Z}_{(q)}}$ completes the proof in the first case.

2) $F\left(\mathbb{Z}_{(p)}, n\right)$. Define $H=\bigoplus_{q \neq p} \mathbb{Z}_{q}$ and $G=\mathbb{Z}_{(p)}$. Then we obtain $n$-dimensional $Y \subset \mathbb{R}^{n+2}$ which is one-dimensional with respect to $\mathbb{Z}_{q^{\infty}}$ and $\mathbb{Z}_{q}$. By virtue of the Bokstein inequality c-dim $\mathbb{Z}_{\mathbb{p}_{p}} Y \leq \max \left\{c-\operatorname{dim}_{\mathbb{Q}} Y, c-\operatorname{dim}_{\mathbb{Z}_{(p)}} Y-1\right\}$ it is sufficient to show that $c-\operatorname{dim}_{\mathbb{Z}_{p} \infty} Y=n$.

Lemma 3.1 implies that $H_{n}(Y ; G) \neq 0$. Therefore $\operatorname{Hom}\left(\check{H}^{n}(Y), G\right) \neq 0[\mathbf{2 3}]$. Hence the group $\check{H}^{n}(Y)$ can not be divisible by $p$. This means that $\check{H}^{n}(Y) \otimes \mathbb{Z}_{p^{\infty}} \neq 0$ and hence $\check{H}^{n}\left(Y ; \mathbb{Z}_{p^{\infty}}\right) \neq 0$.

3) $F\left(\mathbb{Z}_{p}, n\right)$. Define $H=\mathbb{Z}\left[\frac{1}{p}\right]$ and $G=\mathbb{Z}_{p}$. By the Lemma 3.2 we obtain an $n$-dimensional compactum $Y \subset \mathbb{R}^{n+2}$ which is one-dimensional with respect to the groups $\mathbb{Q}, \mathbb{Z}_{(q)}, \mathbb{Z}_{q}, \mathbb{Z}_{q \infty}(q \neq p)$ and $H_{n}\left(Y, \mathbb{Z}_{p}\right) \neq 0$. Since $\operatorname{Hom}\left(\check{H}^{n}(Y), \mathbb{Z}_{p}\right)$ is nontrivial, the product $\ddot{H}^{n}(Y) \otimes \mathbb{Z}_{p}$ is non-trivial and hence $c-\operatorname{dim}_{\mathbb{Z}_{p}} Y=n$. The equality c- $\operatorname{dim}_{\mathbb{Z}_{(p)}} Y=n$ follows by the Bokstein theorem [15] which claims that for a finite dimensional compact space $Y$ there is a prime $p$ such that $\operatorname{dim} Y=c-\operatorname{dim}_{\mathbb{Z}_{(p)}} Y$, and the equality c- $\operatorname{dim}_{\mathbb{Z}_{p} \infty} Y=n-1$ follows from the Bokstein inequalities [15].

4) $F\left(\mathbb{Z}_{p^{\infty}}, n\right)$. Consider $L=M\left(\mathbb{Z}\left[\frac{1}{p}\right], 1\right) \vee M\left(\mathbb{Z}_{p}, n-1\right)$.

First we show that $L * M\left(\mathbb{Z}_{p^{\infty}}, 1\right)$ is an $n+1$-connected space. We have $H_{i}(L *$ $\left.M\left(\mathbb{Z}_{p^{\infty}}, 1\right)\right)=H_{i-1}\left(L \wedge M\left(\mathbb{Z}_{p^{\infty}}, 1\right)\right)=H_{i-1}\left(M\left(\mathbb{Z}\left[\frac{1}{p}\right], 1\right) \wedge M\left(\mathbb{Z}_{p^{\infty}}, 1\right)\right) \oplus H_{i-1}(M$ $\left.\left(\mathbb{Z}_{p}, n-1\right) \wedge M\left(\mathbb{Z}_{p^{\infty}}, 1\right)\right)$. Since $\mathbb{Z}\left[\frac{1}{p}\right] \otimes \mathbb{Z}_{p^{\infty}}=0$ and $\operatorname{Tor}\left(\mathbb{Z}\left[\frac{1}{p}\right], \mathbb{Z}_{p^{\infty}}\right)=0$, it follows that $M\left(\mathbb{Z}\left[\frac{1}{p}\right], 1\right) \wedge M\left(\mathbb{Z}_{p^{\infty}}, 1\right)$ is contractible. Notice that $H_{i-1}\left(M\left(\mathbb{Z}_{p}, n-\right.\right.$ 1) $\left.\wedge M\left(\mathbb{Z}_{p^{\infty}}, 1\right)\right)=0$ for $i-1 \leq n$. Then the Hurewicz theorem implies that $L * M\left(\mathbb{Z}_{p^{\infty}}, 1\right)$ is $n+1$-connected.

Lemma 3.1 implies that there exist an $n$-dimensional compactum $Y \subset \mathbb{R}^{n+2}$ with the property $Y \tau\left(M\left(\mathbb{Z}\left[\frac{1}{p}\right], 1\right) \vee M\left(\mathbb{Z}_{p}, n-1\right)\right)$. Hence we have $Y \tau M\left(\mathbb{Z}\left[\frac{1}{p}\right], 1\right)$ and $Y \tau M\left(\mathbb{Z}_{p}, n-1\right)$. Therefore $c-\operatorname{dim}_{\mathbb{Z}\left[\frac{1}{p}\right]} Y \leq 1$ and $c$ - $\operatorname{dim}_{\mathbb{Z}_{p}} Y \leq n-1$. These inequalities completely define the space $F\left(\mathbb{Z}_{p^{\infty}}, n\right)$.

\section{Proof of the main theorem.}

Theorem 4. Suppose that $X$ and $Y$ are compacta and $\operatorname{dim} X, \operatorname{dim} Y \neq n-2$. Then the following are equivalent

1) $\operatorname{dim} X \times Y<n$,

2) $X \| Y$ in $\mathbb{R}^{n}$.

Proof. 2) $\Rightarrow 1$ ) is contained in [13]. 
1) $\Rightarrow 2$ ). A non-trivial case is $\operatorname{dim} X, \operatorname{dim} Y<n-2$.

Since every compactum $X$ has the cohomological dimension type of a countable union of fundamental compacta (see [15] or [14]), Theorem 3 implies that there is a compactum $X^{\prime} \subset \mathbb{R}^{n}$ with c- $\operatorname{dim}_{G} X=\mathrm{c}$ - $\operatorname{dim}_{G} X^{\prime}$ for all abelian groups $G$.

According to [14] (Lemma 1.2) every map $h: X^{\prime} \rightarrow \mathbb{R}^{n}$ can be $\epsilon$-approximated for given $\epsilon>0$ by a map $h^{\prime}$ with c-dim ${ }_{G} h^{\prime}\left(X^{\prime}\right)=c-\operatorname{dim}_{G} X^{\prime}$ for all $G$.

Since the dimension of the product of compacta is determined by the cohomological dimensions of the factors, we have $\operatorname{dim} h^{\prime}\left(X^{\prime}\right) \times Y<n$.

By the Negligibility Criterion (see introduction) and the Stanko tameness theorem [21] $h^{\prime}\left(X^{\prime}\right)$ is $Y$-negligible.

Since $h^{\prime}$ is an arbitrary approximation for $h$ and $h$ itself is an arbitrary map, we have the property $X^{\prime} \| Y$.

Then by [20], [14] any map $g: Y \rightarrow \mathbb{R}^{n}$ for any $\epsilon>0$ has an $\epsilon$-approximation $g^{\prime}$ such that $g^{\prime}(Y)$ is $X^{\prime}$-negligible.

By the Negligibility Criterion we have the inequality $\operatorname{dim} g^{\prime}(Y) \times X^{\prime}<n$ and hence $\operatorname{dim} g^{\prime}(Y) \times X<n$.

By the Negligibility Criterion and the Stanko tameness theorem $[21] g^{\prime}(Y)$ is $X$-negligible.

Now if we have maps $f: X \rightarrow \mathbb{R}^{n}$ and $g: Y \rightarrow \mathbb{R}^{n}$ and given $\epsilon>0$ we choose $g^{\prime}$ $\epsilon$-close to $g$. Then by the $X$-negligibility of $g^{\prime}(Y)$ there exists $f^{\prime}: X \rightarrow \mathbb{R}^{n} \epsilon$-close to $f$ with $f^{\prime}(X) \cap g^{\prime}(Y)=\emptyset$.

So, $X \| Y$.

Corollary 5. Let $X$ be a compact metric space of the dimension $<n-2$. Then every map of $X$ to $n$-dimensional Euclidean space can be approximated arbitrarily closely by maps with the images of the same cohomological dimension type.

Proof. In [14] it was shown that the positive solution of the Mapping Intersection Problem implies the above statement.

Corollary 6. We may assume that in Theorem 4 the dimension of only one compactum $($ say $X)$ is not equal to $n-2$.

Proof. 2) $\Rightarrow 1$ ) is considered in [13]. 1) $\Rightarrow 2$ ): Let $f: X \rightarrow \mathbb{R}^{n}$ and $g: Y \rightarrow \mathbb{R}^{n}$ be arbitrary maps and $\epsilon>0$ be given. By Corollary 5 we can choose an $\epsilon / 2$ approximation $f^{\prime}: X \rightarrow \mathbb{R}^{n}$ of $f$ with $f^{\prime}(X)$ having the same cohomological dimension type as $X$. By the Stanko tameness theorem [21] there is a tame reimbedding of $f^{\prime}(X)$ in $\mathbb{R}^{n}$ which is $\epsilon / 2$-close to the identity. Since $\operatorname{dim} f^{\prime}(X) \times Y<n$, by the Negligibility Criterion there is an $\epsilon$-approximation $g^{\prime}$ of $g$ avoiding the reimbedded set $f^{\prime}(X)$. This means that the property $X \| Y$ is checked.

I am thankful to E. V. Schepin for pointing out me the last Corollary. Also I am thankful to J. Dydak who informed me about [25] and gave me an elegant proof of its main result. 


\section{References}

[1] D. McCullough and L. Rubin, Some m-dimensional compacta admitting a dense set of imbeddings into $\mathbb{R}^{2 m}$, Fund. Math., 133 (1989), 237-245.

[2] J. Krasinkiewicz, Imbeddings into $\mathbb{R}^{n}$ and dimension of products, Fund. Math., 133 (1989), 247-253.

[3] S. Spiez, Imbeddings in $\mathbb{R}^{2 m}$ of $m$-dimensional compacta with $\operatorname{dim} X \times X<2 m$, Fund. Math., 134 (1990), 105-115.

[4] - On pairs of compacta with $\operatorname{dim}(X \times Y)<\operatorname{dim} X+\operatorname{dim} Y$, Fund. Math., 135 (1990), 213-222.

[5] A.N. Dranishnikov and E.V. Schepin, Stable intersection of compacta in euclidean space, Russian Math. Surveys, 44(5) (1989), 194-195.

[6] A.N. Dranishnikov, D. Repovs and E.V. Schepin, On intersections of compacta of complementary dimensions in Euclidean space, Topol. Appl., 38 (1991), 237-253.

[7] A. Dranishnikov, Homological dimension theory, Russian Math. Survey, 43(4) (1988), 11-63.

[8] , Spanier-Whitehead duality and stability of intersections of compacta, Trudy Steklov Math. Inst., 196 (1991), 47-50.

[9] A.N. Dranishnikov, D. Repovs and E.V. Schepin, On intersection of compacta in euclidean space: the metastable case, Tsukuba J. Math., 17(2) (1993).

[10] S. Spiez and H. Torunczyk, Moving compacta in $\mathbb{R}^{n}$ apart, Topol. Appl., 41 (1991), 193-204.

[11] A.N. Dranishnikov, On intersection of compacta in Euclidean space, Proc. Amer. Math. Soc., 112 (1991), 267-275.

[12] On intersection of compacta in Euclidean space. II, Proc. Amer. Math. Soc., 113 (1991), 1149-1154.

[13] A.N.Dranishnikov and J.West, On compacta that intersect unstably in Euclidean space, Topol. Appl., 43 (1992), 181-187.

[14] A.N. Dranishnikov, D. Repovs and E.V.Schepin, On approximation and embedding problems for cohomological dimension, Topol. Appl., 55 (1994), 67-86.

[15] V.I. Kuzminov, Homological dimension theory, Russian Math. Surveys, 23(5) (1968), 1-45.

[16] J. Dydak, Cohomological dimension and metrizable spaces II, preprint.

[17] A.N. Dranishnikov, D. Repovs and E.V. Schepin, On the failure of the UrysohnMenger formula for cohomological dimension, Proc. Amer. Math. Soc., to appear.

[18] A.N. Dranishnikov, An extension of mappings into $C W$-complexes, Math. of the USSR-Sbornik, 74(1) (1993), 47-56.

[19] A. Dold and R. Thom, Quasifaserungen und unedliche symmetrische Produkte, Ann. of Math., 67(2) (1958), 239-281.

[20] J. Luukkainen, Embeddings of n-dimensional locally compact metric spaces to $2 n$ manifolds, Math. Scand., 68 (1991), 193-209.

[21] M.A. Stanko, Embeddings of compacta in Euclidean space, Math. USSR-Sbornik, 10 (1970), 234-254.

[22] S. Eilenberg, Un theorem de la dualite, Fund. Math, 26 (1936), 280-282. 
[23] W.S. Massey, Homology and cohomology theory, Marcel Dekker, Inc., New York and Basel, 1978.

[24] E. Spanier, Algebraic topology, McGraw-Hill, 1966.

[25] W. Olszewski, Completion theorem for cohomological dimensions, preprint, 1993.

Received April 27, 1993 and revised March 14, 1994.

UNIVERSITY OF MIAMI

Coral Gables, FL 33124

AND

Steklov Mathematical Institute

Moscow, Russia

E-mail address: dranish@math.miami.edu 
Peng Lin and Richard Rochberg, Trace ideal criteria for Toeplitz and Hankel operators on the weighted Bergman spaces with exponential type

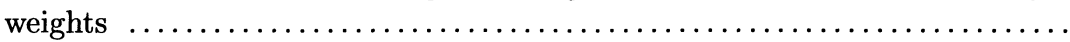

Donald E. Marshall and Arne Stray, Interpolating Blaschke products . .

Kathy D. Merrill and Lynne $\mathbf{H}$. Walling, On quadratic reciprocity over

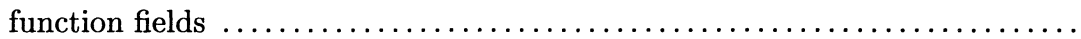

Takahiko Nakazi and Masahiro Yamada, $\left(A_{2}\right)$-conditions and Carleson

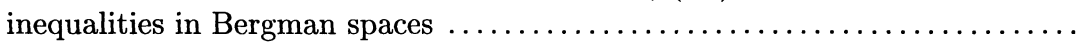

C. Ott, A note on a paper of E. Boasso and A. Larotonda ..............

Victor Patrangenaru, Classifying 3 and 4 dimensional homogeneous Riemannian manifolds by Cartan triples

Carlo Pensavalle and Tim Steger, Tensor products with anisotropic principal series representations of free groups

Ying Shen, On Ricci deformation of a Riemannian metric on manifold with boundary

Albert Jeu-Liang Sheu, The Weyl quantization of Poisson $S U(2) \ldots \ldots$

Alexandra Shlapentokh, Polynomials with a given discriminant over fields of algebraic functions of positive characteristic

Eric Stade and D.I. Wallace, Weyl's law for $S L(3, \mathbb{Z}) \backslash S L(3, \mathbb{R}) / S O(3, \mathbb{R})$

Christopher W. Stark, Resolutions modeled on ternary trees ........ 557

Per Tomter, Minimal hyperspheres in two-point homogeneous spaces .....

Jun Tomiyama, Topological Full groups and structure of normalizers in

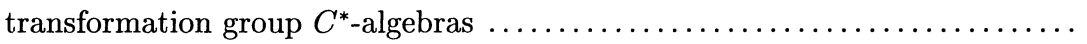

Nik Weaver, Subalgebras of little Lipschitz algebras 


\section{PACIFIC JOURNAL OF MATHEMATICS}

\section{Volume $173 \quad$ No. $2 \quad$ April 1996}

A mean value inequality with applications to Bergman space operators

PATRICK ROBERT AHERn and ZELJKO CUCKOVIC

$H^{p}$-estimates of holomorphic division formulas

MATS ANDERSSON and HASSE CARLSSON

Group structure and maximal division for cubic recursions with a double root

Christian JeAn-Claude Ballot

The Weil representation and Gauss sums

ANTONiA WiLson BLUHER

Duality for the quantum $E(2)$ group

ALFONS VAN DAELE and S. L. WORONOWICZ

Cohomology complex projective space with degree one codimension-two fixed submanifolds 387

KARL HEINZ DOVERMANN and ROBERT D. LITTLE

On the mapping intersection problem

ALEXANDER DRANISHNIKOV

From the $L^{1}$ norms of the complex heat kernels to a Hörmander multiplier theorem for

sub-Laplacians on nilpotent Lie groups

\section{XUAN THINH DUONG}

Isoperimetric inequalities for automorphism groups of free groups

Allen E. Hatcher and Karen Vogtmann

Approximation by normal elements with finite spectra in $C^{*}$-algebras of real rank zero

HUAXIN LIN

Interpolating Blaschke products

DonALD EdDY MARShall and ARNE STRAY

Interpolating Blaschke products generate $H^{\infty}$

JOHN BRADY GARNETT and ARTUR NICOLAU

Classifying 3- and 4-dimensional homogeneous Riemannian manifolds by Cartan triples

VICTOR PATRANGENARU

Polynomials with a given discriminant over fields of algebraic functions of positive

characteristic

ALEXANDRA SHLAPENTOKH

Resolutions modeled on ternary trees

CHRISTOPHER W. STARK

Topological full groups and structure of normalizers in transformation group $C^{*}$-algebras 